\section{The diagnostic ability of an additional midline peripheral zone biopsy in transrectal ultrasonography-guided} 12-core prostate biopsy to detect midline prostate cancer

\author{
Inpyeong Hwang, Sang Youn Kim, Jeong Yeon Cho, Myoung Seok Lee, Seung Hyup Kim
}

Department of Radiology, Seoul National University Hospital and Institute of Radiation

Medicine, Seoul National University Medical Research Center, Seoul, Korea

Purpose: The goal of this study was to evaluate the diagnostic effect of adding a midline peripheral zone (PZ) biopsy to the 12-core biopsy protocol used to diagnose prostate cancer (PC), and to assess the clinical and pathologic characteristics of midline-positive PC in order to identify a potential subgroup of patients who would require midline PZ biopsy.

Methods: This study included 741 consecutive patients who underwent a transrectal ultrasonography-guided, 12-core prostate biopsy with an additional midline core biopsy between October 2012 and December 2013. We grouped patients by the presence or absence of PC and subdivided patients with PC based on the involvement of the midline core. The clinical characteristics of these groups were compared, including serum prostate-specific antigen (PSA) concentrations, PSA density, and pathological features in the biopsy specimens.

Results: PC was detected in 289 patients (39.0\%). Among the PC patients, 66 patients (22.8\%) had midline PC. No patients were diagnosed with PC based only on a midline core. The Gleason scores, number of positive cores, tumor core length, serum PSA concentrations, and PSA density were significantly higher in patients with midline-positive $P C(P<0.001)$. Furthermore, significant cancer was more frequent in the midline-positive group (98.5\% vs. $78.0 \%)$.

Conclusion: Patients showing a positive result for $\mathrm{PC}$ in a midline $\mathrm{PZ}$ biopsy were more likely to have multiple tumors or large-volume PC with a high tumor burden. However, our data indicated that an additional midline core biopsy is unlikely to be helpful in detecting occult midline PC.

Keywords: Prostatic neoplasms; Image-guided biopsy; Ultrasonography

\section{Introduction}

Transrectal ultrasonography (TRUS)-guided biopsy is the standard approach for the histopathologic diagnosis of prostate cancer (PC). The best predictor of the aggressiveness of prostate adenocarcinoma is the Gleason score, which can only be obtained through the histopathologic

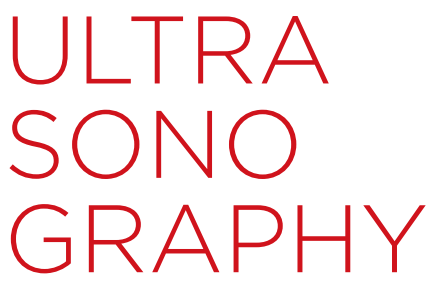

ORIGINAL ARTICLE

http://dx.doi.org/10.14366/usg. 15039 pISSN: 2288-5919 - elSSN: 2288-5943 Ultrasonography 2016;35:61-68

Received: June 29, 2015

Revised: August 17, 2015

Accepted: August 18, 2015

Correspondence to:

Sang Youn Kim, MD, Department of Radiology, Seoul National University Hospital and Institute of Radiation Medicine, Seoul National University Medical Research Center, 101 Daehakro, Jongno-gu, Seoul 03080, Korea

Tel. +82-2-2072-4897

Fax. $+82-2-743-6385$

E-mail: iwishluv@empas.com

This is an Open Access article distributed under the terms of the Creative Commons Attribution NonCommercial License (http://creativecommons.org/ licenses/by-nc/3.0/) which permits unrestricted noncommercial use, distribution, and reproduction in any medium, provided the original work is properly cited.

Copyright $(2016$ Korean Society of Ultrasound in Medicine (KSUM)

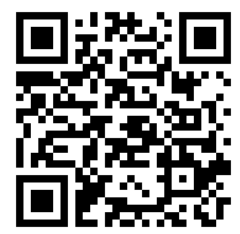

How to cite this article:

Hwang I, Kim SY, Cho JY, Lee MS, Kim SH. The diagnostic ability of an additional midline peripheral zone biopsy in transrectal ultrasonography-guided 12-core prostate biopsy to detect midline prostate cancer. Ultrasonography. 2016 Jan;35(1):61-68. 
analysis of biopsy samples. Thus, prostate biopsy, usually performed with a TRUS-guided needle, remains an essential component of the diagnostic work-up. However, TRUS-guided prostate biopsies are usually performed as systematic random biopsies, in which, for example, six cores are obtained from the paramedian peripheral zone (PZ) [1]. In order to enhance the detection of PC, extended techniques in which 12-core specimens are obtained are the currently accepted standard protocol. The specimens include a sextant biopsy and additional cores from the lateral prostate to allow sampling of the more lateral PZ $[2,3]$. Despite these modifications, which require obtaining additional cores, the rate of false-negatives remains substantial.

Biopsy of the midline PZ is controversial. In the routine protocol, no cores are acquired from the midline PZ. One study using a computer simulation advocated midline biopsies [4]. Another published study reported that 10 additional cancers among 425 patients $(2.3 \%)$ were detected by including three midline $\mathrm{PZ}$ core biopsies in an 18-core prostate biopsy protocol that included the transition zone [5]. Although the midline PZ makes up a relatively small portion of the volume of the prostate, cancer can develop in the midline $\mathrm{PZ}$ and it is possible for the extended 12-core systematic random biopsy scheme to miss such cases of cancer. Acquiring one additional midline core in the mid-to-basal portion could be easily incorporated into the extended 12-core biopsy without significantly increasing the procedure time. TRUS can easily visualize the midline sagittal plane of the prostate and provide excellent guidance in order to avoid urethral injury during the biopsy procedure.

It has been reported that prostate-specific antigen (PSA) density is a more effective screening parameter for PC than serum PSA concentration alone [6]. We hypothesized that an additional midline PZ biopsy might be beneficial in specific subgroups of patients, such as those with a high PSA density.

The purpose of our study was to evaluate the PC detection performance of a midline PZ biopsy performed in addition to the standard 12-core biopsy protocol, and to assess the clinical and pathologic characteristics of midline-positive PC in order to identify a potential subgroup of patients who would benefit from midline PZ biopsies.

\section{Materials and Methods}

The institutional review board of our institution approved this retrospective study and the requirement for informed consent was waived.

\section{Patient Selection}

Between October 2012 and December 2013, 760 patients were referred from the Department of Urology in our institution for further evaluation of suspected PC based on an abnormal digital rectal examination and/or an elevated serum PSA concentration. During the study period, 764 TRUS-guided biopsies were performed on those patients. Extended 12-core systematic random biopsies with an additional midline basal PZ biopsy were routinely performed in our institution in consensus with the Department of Urology. We excluded 18 patients for the following reasons: the use of a sextant biopsy scheme $(n=3)$, a missing midline basal PZ biopsy $(n=6)$, the presence of malignant tumors other than prostate adenocarcinoma $(n=3)$, and the diagnosis of atypical small acinar proliferation $(n=7)$.

Four patients underwent two TRUS-guided biopsies during the study period. One patient was initially classified as having atypical small acinar proliferation and high-grade prostatic intraepithelial neoplasia in one core, while a subsequent repeated biopsy revealed no carcinoma. Since it was possible that the second biopsy resulted in a false-negative, this case was classified as atypical small acinar proliferation and excluded from further evaluation. Another patient underwent two biopsies that were positive for prostate adenocarcinoma: a biopsy resulting in the initial diagnosis and a 1-year follow-up biopsy during hormonal therapy. The other two patients were negative for prostate adenocarcinoma on two biopsies. For these three patients who underwent two biopsies, the initial biopsy results and clinical characteristics were included in the analysis.

Finally, a total of 741 biopsy results from 741 patients comprised the population of our study.

\section{TRUS-Guided Biopsy}

TRUS-guided biopsy was performed by two genitourinary radiologists (J.Y.C. and S.Y.K.) with two ultrasound systems (Acuson Sequoia, Siemens Healthcare, Erlangen, Germany, and iU22, Philips Healthcare, Best, The Netherlands). The probes used were 10-5 $\mathrm{MHz}$ or $9-5 \mathrm{MHz}$ broadband curved-array endorectal probes.

Bowel cleansing was carried out through the oral administration of $10 \mathrm{mg}$ of bisacodyl one day before the biopsy and the administration of two bisacodyl suppositories the morning of the biopsy. Antiplatelet agents were discontinued seven days before the procedure. Prophylactic oral cephalosporin (100 mg of cefixime) was administered two times per day 1 day before the biopsy and continued for 5 days. Intravenous cephalosporin ( $1 \mathrm{~g}$ of ceftriaxone) was administered 1 hour before the procedure.

Imaging was performed in the axial and sagittal planes from the seminal vesicles to the apex of the prostate gland, including gray-scale and color Doppler. After volume measurement and routine imaging, the prostate was divided into 13 sectors for biopsy (Fig. 1A). For local anesthesia, $5 \mathrm{~mL}$ of $2 \%$ lidocaine was 
administered into a bilateral neurovascular bundle. An extended 12core systematic random biopsy was performed using an automatic spring-loaded gun biopsy device with an 18-gauge needle (Acecut, TSK Laboratory, Tochigi, Japan); six paramedian cores and six lateraldirected cores were obtained from the paramedian and the lateral apical, middle, and basal PZs, respectively. The additional midline biopsy was taken at the midline basal PZ in the sagittal plane (Fig. 1B). If a focal lesion was identified on gray-scale or color Doppler imaging, an additional targeted biopsy was taken and included in the pathologic evaluation.

\section{Assessment of Clinical and Pathologic Characteristics}

The clinical and pathological characteristics of the patients and the tumors were obtained from a retrospective review of electronic medical records, radiologic reports, and pathologic reports. We obtained information about the patients' age, total prostate volume, transition zone volume, serum PSA concentration, and PSA density. Serum PSA concentration values were obtained within 1 month prior to the biopsy procedure. Total prostate volume was measured on the TRUS using the following equation: prostate volume $(m L)=\pi / 6 \times D_{1} \times D_{2} \times D_{3}\left(D_{n}\right.$, prostate diameter measured by ultrasound on one of three orthogonal planes). Transition zone volume was also measured in the same manner, based on measurements of the diameter of the transition zone in three orthogonal planes.

An experienced genitourinary pathologist at our institution (K.C.M.) provided pathology reports for the prostate core biopsy specimens. When a biopsy specimen was classified as prostate adenocarcinoma, the Gleason score (primary+secondary pattern), tumor length, and core length were reported for each core [7]. The pathological characteristics, including the maximum Gleason score from each core, the number of positive cores, the sum of the tumor length, and the core length ratio (sum of tumor length/sum of core length) were analyzed. If the patient underwent a targeted biopsy, the targeted core was included in the analysis. In addition, the presence of significant PC was investigated. Significant PC was defined as any cancer with a core length of more than $3 \mathrm{~mm}$ in any of the 12 cores or any Gleason pattern more than 3 according to the Harnden criteria [8].

In most patients who were diagnosed with $\mathrm{PC}$, the presence of distant metastases was assessed by using magnetic resonance imaging, bone scintigraphy, and/or ${ }^{18} \mathrm{~F}$-fluorodeoxyglucose positron emission tomography. If the patient underwent radical prostatectomy, an experienced genitourinary pathologist (K.C.M.) also evaluated the surgical prostatectomy specimen. We obtained the Gleason score, tumor volume ratio (approximate tumor volume relative to the volume of the entire prostate), multicentricity of $P C$, and the presence of capsular invasion or seminal vesicle invasion from the pathology report.

\section{Statistical Analysis}

Patients were divided into two groups depending on whether their pathologic results were positive or negative for $\mathrm{PC}$. The prevalence of $P C$ and midline-positive PC was calculated. The positive PC group was further subdivided based on the presence of PC in the midline
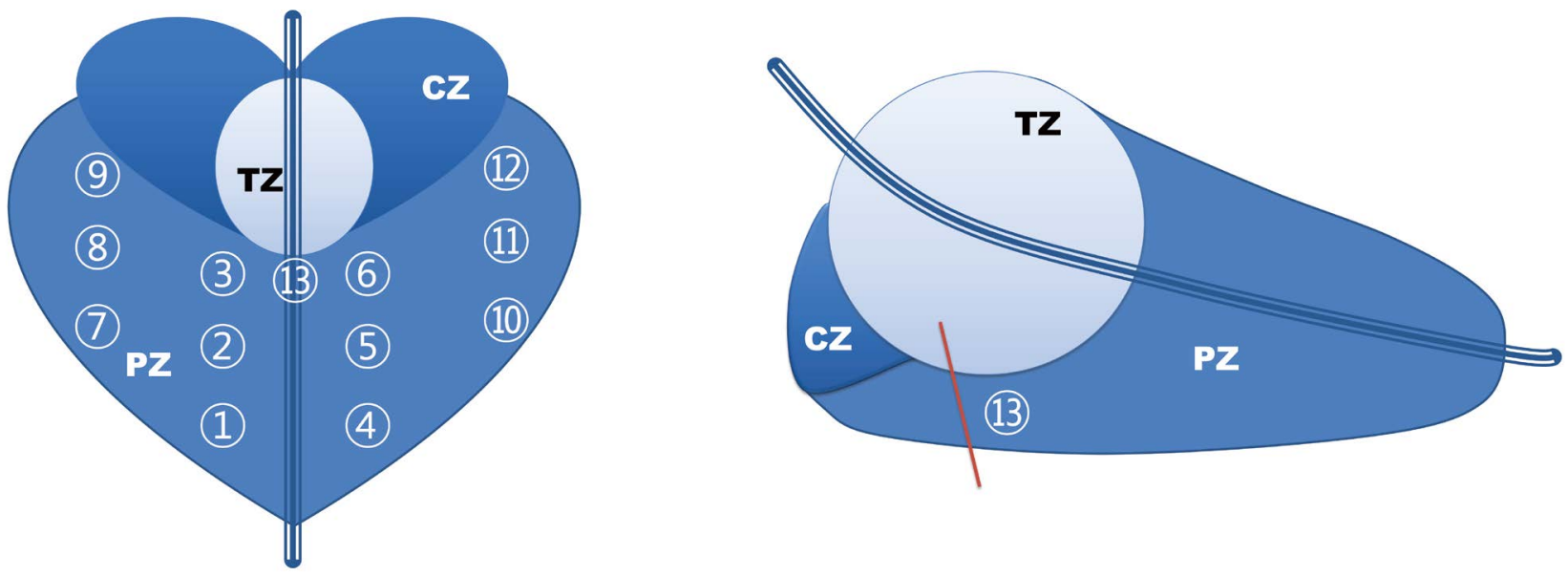

A

B

Fig. 1. Schematic diagram.

The diagrams show a coronal (A) and a midline sagittal (B) scan of the prostate gland for the performance of a midline peripheral zone biopsy. 1-3, right paramedian peripheral zone; 4-6, left paramedian peripheral zone; 7-9, right lateral peripheral zone; 10-12, left lateral peripheral zone; 13, midline basal peripheral zone. The red line represents the direction of the biopsy needle. $C Z$, central zone; PZ, peripheral zone; TZ, transition zone. 
core sample. Subgroup analysis was performed according to prostate volume, PSA concentration, and PSA density. The prevalence of $P C$ and midline PC was investigated in each subgroup. In order to evaluate the diagnostic performance of PSA density for the detection of $P C$, we analyzed the receiver operating characteristics (ROC) curve reflecting the ability of total prostate volume, transition zone volume, PSA concentration, and PSA density to predict the presence of $P C$ and midline $P C$. We determined the optimal cutoff point for each variable by using the Youden index, and the sensitivity and specificity of each variable were calculated.

The statistical analyses were conducted using IBM SPSS Statistics ver. 19.0 (IBM Corp., Armonk, NY, USA). Two-tailed P-values of $<0.05$ were considered to indicate statistical significance in all statistical analyses. We compared the clinical characteristics of the positive and negative PC groups using the Student's t test. In addition, the clinical and pathologic characteristics were compared between the midline-positive and midline-negative groups, using the Student's $t$ test for continuous variables and Fisher exact test for binary variables.

\section{Results}

\section{Prevalence of PC and Midline-Positive PC}

Among the 741 patients included in our study, 289 (39.0\%) were positive for $\mathrm{PC}$. The $\mathrm{PC}$-positive patients were found to be older, to have a lower total prostate volume, and to have a lower transition zone volume (Table 1). The serum PSA concentrations and PSA density were higher in the PC-positive group (Table 1). A target biopsy for a ultrasonography-demonstrated focal lesion was performed in 191 patients, of whom 78 (40.8\%) were diagnosed with PC as a result of the targeted core biopsy. Among the PC-positive patients, $66(22.8 \%)$ were diagnosed with $\mathrm{PC}$ in the midline PZ core biopsy. No patient had only a positive midline PZ core biopsy, and most of the midline-positive patients showed at least one or more positive paramedian cores. Only two patients $(0.26 \%)$ were diagnosed with $\mathrm{PC}$ in a midline $\mathrm{PZ}$ core with a negative $\mathrm{PC}$ result in the paramedian

Table 1. Clinical characteristics of the study population

\begin{tabular}{lccc}
\hline \multicolumn{1}{c}{ Characteristic } & $\begin{array}{c}\text { Positive for } \\
\text { PC }(\mathrm{n}=289)\end{array}$ & $\begin{array}{c}\text { Negative for } \\
\mathrm{PC}(\mathrm{n}=452)\end{array}$ & P-value \\
\hline Age $(\mathrm{yr})$ & $68.8 \pm 7.6$ & $63.7 \pm 8.6$ & $<0.001$ \\
Prostate volume $(\mathrm{mL})$ & $48.4 \pm 22.2$ & $63.7 \pm 32.6$ & $<0.001$ \\
Transition zone volume $(\mathrm{mL})$ & $25.2 \pm 17.6$ & $37.5 \pm 26.9$ & $<0.001$ \\
Serum PSA concentration $(\mathrm{ng} / \mathrm{mL})$ & $70.2 \pm 292$ & $7.22 \pm 10.6$ & $<0.001$ \\
PSA density $\left(\mathrm{ng} / \mathrm{mL}^{2}\right)$ & $1.24 \pm 5.09$ & $0.139 \pm 0.384$ & $<0.001$ \\
\hline
\end{tabular}

Values are presented as mean $\pm S D$ or number.

PC, prostate cancer; PSA, prostate-specific antigen. cores. In one patient, the biopsy revealed PC in the right mid lateral $\mathrm{PZ}$ core and the midline PZ core. The patient underwent radical prostatectomy. The prostatectomy specimen revealed that the dominant tumor was located in the right apico-mid lateral area with a Gleason score of $7(3+4)$, and the tumor volume comprised $5 \%$ of the prostate volume. Another tumor was located in the midline basal portion. No PC was detected in the paramedian area. In the other patient, an ill-defined low echoic lesion was found in the left mid-base paramedian PZ on TRUS, and a targeted biopsy was also obtained. In the 12-core systematic random biopsy, PC was only detected in the left apical lateral PZ core. The midline PZ core and the target core directed toward the left mid-base paramedian PZ revealed the presence of $\mathrm{PC}$. This patient also underwent a radical prostatectomy. Multicentric tumors were present in the bilateral mid and basal PZ and the midline PZ, with a Gleason score of 7 $(3+4)$, and the tumor comprised $3 \%$ of the volume of the radical prostatectomy specimen.

\section{Comparison of the Clinical and Pathologic Characteristics between Midline-Positive and Midline-Negative PC}

Multiple tumors and large-volume PC were more frequent in the midline-positive group to a statistically significant extent (average number of positive cores, 9.6 vs. 3.4, $\mathrm{P}<0.001$ ) (Table 2). The midline-positive group showed higher Gleason scores (8.0 vs. $6.9, P<0.001)$, a higher total length of the tumor $(6.8 \mathrm{~mm}$ vs. $1.4 \mathrm{~mm}, \mathrm{P}<0.001)$, and a higher core length ratio $(61.3 \% \mathrm{vs}$. $35.5 \%, P<0.001)$ when compared to the midline-negative group. Furthermore, the prevalence of significant PC was higher in the midline-positive group (98.5\% [65/66]) than in the midline-negative group (78.0\% [174/223]). In terms of clinical characteristics, the serum PSA concentrations and PSA density were significantly different in the midline-positive and midline-negative groups, while the prostate volume and the volume of the transition zone were not significantly different between the two groups (Table 2).

Of the 289 patients who were diagnosed with PC, 279 patients underwent imaging studies to evaluate the extent of the disease. In 37 patients $(9.7 \%)$, the imaging studies found evidence of distant metastases. A significant difference was found between the midlinepositive and midline-negative groups regarding the presence of distant metastases: 28 of the 64 patients $(43.8 \%)$ in the midlinepositive group and nine of the 215 patients $(4.2 \%)$ in the midlinenegative group $(\mathrm{P}<0.001)$ had distant metastases.

A total of 156 patients underwent radical prostatectomy. The Gleason score and tumor volume/prostate volume ratio of the surgical specimens were significantly higher in the midline-positive group $(\mathrm{P}<0.001)$, and capsular invasion or seminal vesicle invasion were detected significantly more frequently in the midline-positive 
Table 2. Clinical and pathologic characteristics of prostate cancer-positive patients: comparison between the midline-positive and midline-negative groups

\begin{tabular}{|c|c|c|c|}
\hline Characteristic & Midline-positive $(n=66)$ & Midline-negative $(n=223)$ & P-value \\
\hline \multicolumn{4}{|l|}{ Clinical characteristic } \\
\hline Age (yr) & $68.2 \pm 8.4$ & $69.0 \pm 7.4$ & 0.482 \\
\hline Prostate volume (mL) & $50.0 \pm 20.2$ & $47.9 \pm 22.8$ & 0.513 \\
\hline Transition zone volume (mL) & $24.6 \pm 14.7$ & $25.3 \pm 18.4$ & 0.766 \\
\hline Serum PSA concentration (ng/mL) & $165.2 \pm 344.5$ & $42.0 \pm 269.5$ & 0.009 \\
\hline PSA density $\left(n g / m L^{2}\right)$ & $3.16 \pm 6.49$ & $0.67 \pm 4.45$ & 0.005 \\
\hline \multicolumn{4}{|l|}{ Pathologic characteristic of biopsy specimens } \\
\hline Maximum Gleason score & $8.0 \pm 1.0$ & $6.9 \pm 0.9$ & $<0.001$ \\
\hline Number of positive cores & $9.6 \pm 3.3$ & $3.4 \pm 2.3$ & $<0.001$ \\
\hline Total tumor length (mm) & $6.8 \pm 3.9$ & $1.4 \pm 1.6$ & $<0.001$ \\
\hline Core length ratio (\%) & $60.5 \pm 19.7$ & $34.1 \pm 19.7$ & $<0.001$ \\
\hline Significant cancer, $\mathrm{n}(\%)^{\mathrm{a})}$ & $65(98.5)$ & $174(78.0)$ & $<0.001$ \\
\hline \multicolumn{4}{|l|}{ Pathologic characteristic of surgical specimens } \\
\hline Number & 26 & 132 & \\
\hline Gleason score & $7.5 \pm 0.9$ & $6.8 \pm 0.6$ & 0.002 \\
\hline Tumor volume ratio (\%) & $43.7 \pm 27.4$ & $12.6 \pm 11.7$ & $<0.001$ \\
\hline Multicentricity, $\mathrm{n}(\%)^{\mathrm{a})}$ & $13(50.0)$ & $92(69.7)$ & 0.069 \\
\hline Capsular or seminal vesicle invasion, $\mathrm{n}(\%)^{\mathrm{a})}$ & $22(84.6)$ & $49(37.1)$ & $<0.001$ \\
\hline
\end{tabular}

Values are presented as mean \pm SD or number.

PSA, prostate-specific antigen.

${ }^{a}$ Number of patients followed by prevalence in parentheses.

group $(\mathrm{P}<0.001)$ (Table 2).

Subgroup Analysis and ROC Curve Analysis of Clinical Characteristics in the Detection of PC and Midline-Positive PC In subgroup analyses according to prostate volume, serum PSA concentrations, and PSA density, the prevalence of PC was higher in patients with lower prostate volume, higher serum PSA concentrations, and greater PSA density. The prevalence of positive-midline PZ core biopsies also increased with serum PSA concentration and PSA density, while patients with a moderate prostate volume of $30-60 \mathrm{~mL}$ had the highest prevalence of positive midline PZ core biopsies (Table 3).

The ROC curve analysis indicated that a higher PSA density was the most predictive clinical variable for the presence of PC (sensitivity, $67.5 \%$; specificity, $79.2 \%$; cutoff value, $0.15 \mathrm{ng} / \mathrm{mL}^{2}$ ) (Table 4, Fig. 2). Higher values of serum PSA concentration and PSA density were predictive of positive midline PZ cores (Table 4); PSA density also had the highest predictive value for positive midline $\mathrm{PZ}$ core biopsies (sensitivity, $81.8 \%$; specificity, $86.9 \%$; cutoff value, $0.26 \mathrm{ng} / \mathrm{mL}^{2}$ ).

\section{Discussion}

The classic prostate sampling technique is the sextant scheme, in which six core samples are obtained. However, this technique has been found to have limited sensitivity for the detection of PC [1]. In order to enhance the detection of $P C$, the incorporation of an additional six cores, targeting each lateral $\mathrm{PZ}$, is now considered the standard protocol $[2,3]$. One study reported that the 12-core extended biopsy scheme, which incorporates additional laterally directed cores, was able to detect $31 \%$ more cases of cancer [3]. However, the routine 12-core biopsy may fail to sample the anterior transition zone, the midline $\mathrm{PZ}$, and the lower portion of the anterior horn of the $\mathrm{PZ}$ due to the limited biopsy needle length and/or the directions of the biopsy. However, increasing the number of biopsy cores to more than 12 cores is not routinely recommended, because doing so does not improve the detection of PC [9]. Several studies have investigated the role of midline PZ biopsies. One study suggested that adding a midline needle biopsy would be beneficial, on the basis of computer modeling [4]. Most studies have reported that the addition of a midline $\mathrm{PZ}$ biopsy only results in a limited additional ability to detect cancer $[2,10,11]$. However, those studies evaluated the addition of a midline biopsy to a sextant biopsy 
scheme. Guichard et al. [5] showed that 10 additional cancers in 425 patients (2.3\%) were detected by the addition of a midline PZ biopsy to an extended prostate biopsy that included the transition zone. Their results were similar to those of previous studies that did not support the addition of a midline PZ biopsy. In our study, 66 patients were positive for midline $P C$, but no patient showed only positive midline PC results, even though our study population for midline core biopsies was larger than those included in previous studies. We evaluated the incorporation of one additional midline core biopsy into an extended 12-core biopsy scheme, whereas Guichard et al. [5] evaluated three additional midline core biopsies in an extended 18-core biopsy scheme. All midline-positive patients in our study showed at least one or more positive sextant cores, with the exception of two patients. Retrospectively, the patients who had positive midline cores tended to show a greater number of cancer-positive cores, higher Gleason scores, greater tumor length, and a higher prevalence of significant cancer. In addition, distant metastases were more frequent, and the tumor volume in surgical

Table 3. Subgroup analysis

\begin{tabular}{|c|c|c|c|}
\hline & All patients $(n=741)$ & Positive for PC $(n=289)^{a)}$ & Midline-positive $(n=66)^{b)}$ \\
\hline \multicolumn{4}{|c|}{ Prostate volume $(\mathrm{mL})$} \\
\hline$<30$ & 72 & $38(52.8)$ & $3(7.9)$ \\
\hline $30-60$ & 402 & $185(46.0)$ & $48(25.9)$ \\
\hline $60-100$ & 205 & $56(27.3)$ & $13(23.2)$ \\
\hline$\geq 100$ & 62 & $10(16.1)$ & $2(20)$ \\
\hline \multicolumn{4}{|c|}{ Serum PSA concentration (ng/mL) } \\
\hline$<4$ & 150 & $33(22.0)$ & $2(6.1)$ \\
\hline $4-10$ & 386 & $118(30.6)$ & $8(6.8)$ \\
\hline $10-20$ & 114 & $62(54.4)$ & $14(22.6)$ \\
\hline$\geq 20$ & 90 & $76(84.4)$ & $42(55.3)$ \\
\hline \multicolumn{4}{|c|}{ PSA density $\left(\mathrm{ng} / \mathrm{mL}^{2}\right)$} \\
\hline$<0.1$ & 288 & 45 (15.6) & $2(4.4)$ \\
\hline $0.1-0.2$ & 247 & $91(36.8)$ & $8(8.8)$ \\
\hline $0.2-0.3$ & 81 & $45(55.6)$ & $5(11.1)$ \\
\hline$\geq 0.3$ & 124 & $108(87.1)$ & $51(47.2)$ \\
\hline
\end{tabular}

Values are presented as number (\%).

Midline- positive only: $n=0$.

PC, prostate cancer; PSA, prostate-specific antigen.

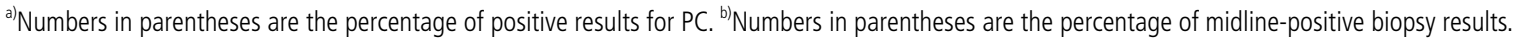

Table 4. ROC analysis comparing the predictive ability of clinical variables to detect prostate cancer and midline-positive prostate cancer

\begin{tabular}{|c|c|c|c|c|c|}
\hline & AUC & $95 \% \mathrm{Cl}$ & Cutoff value & Sensitivity (\%) & Specificity (\%) \\
\hline \multicolumn{6}{|l|}{ Positive for prostate cancer } \\
\hline Prostate volume & 0.663 & $0.624-0.702$ & $<51.8 \mathrm{~mL}$ & 68.9 & 57.5 \\
\hline Transition zone volume & 0.666 & $0.626-0.705$ & $<23.4 \mathrm{~mL}$ & 61.7 & 66.6 \\
\hline PSA & 0.704 & $0.665-0.744$ & $>8.99 \mathrm{ng} / \mathrm{mL}$ & 54.0 & 82.3 \\
\hline PSA density & 0.797 & $0.764-0.831$ & $>0.15 \mathrm{ng} / \mathrm{mL}^{2}$ & 67.5 & 79.2 \\
\hline \multicolumn{6}{|c|}{ Positive midline peripheral zone biopsy } \\
\hline Prostate volume & 0.576 & $0.514-0.638$ & $<50.0 \mathrm{~mL}$ & 66.7 & 52.9 \\
\hline Transition zone volume & 0.604 & $0.543-0.665$ & $<32.0 \mathrm{~mL}$ & 84.4 & 39.3 \\
\hline PSA & 0.881 & $0.833-0.929$ & $>12.8 \mathrm{ng} / \mathrm{mL}$ & 78.8 & 86.8 \\
\hline PSA density & 0.898 & $0.854-0.942$ & $>0.26 \mathrm{ng} / \mathrm{mL}^{2}$ & 81.8 & 86.9 \\
\hline
\end{tabular}

ROC, receiver operating characteristic; AUC, area under the curve; $\mathrm{Cl}$, confidence interval; PSA, prostate-specific antigen. 


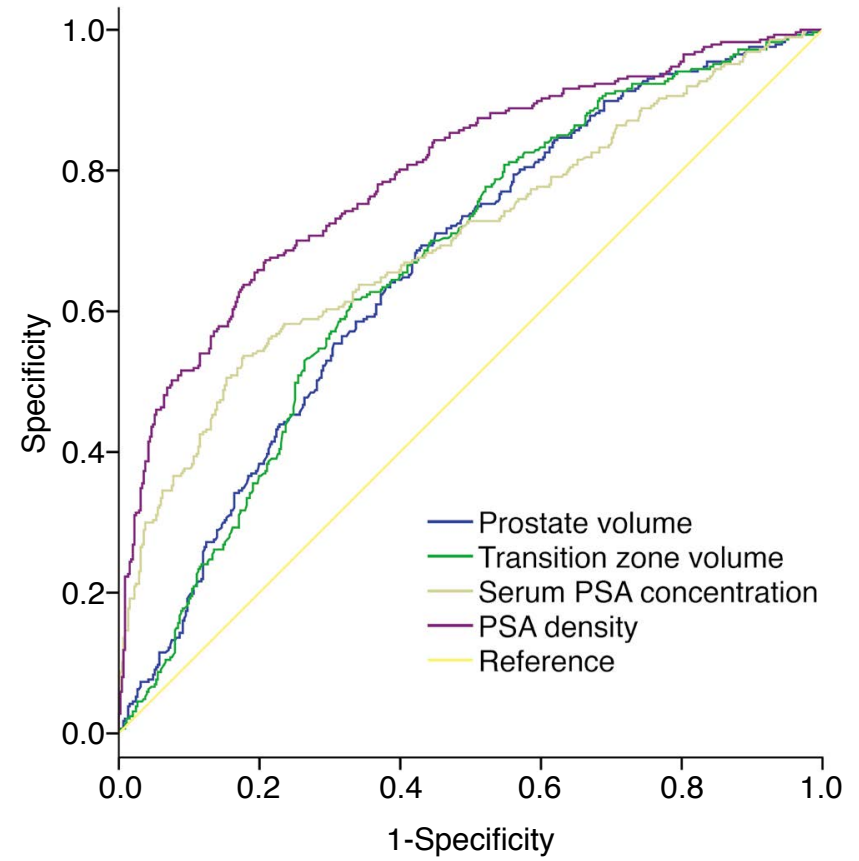

A

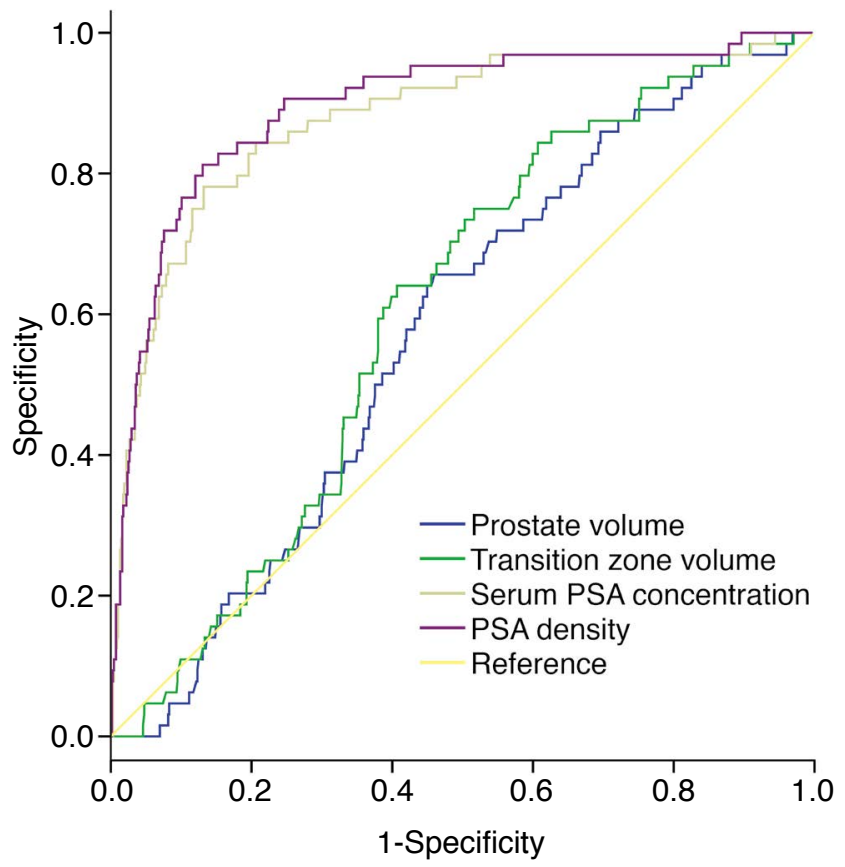

B

Fig. 2. Receiver operating characteristics (ROC) curve.

The predictive abilities of clinical variables for the presence of prostate cancer $(P C)(A)$ and the presence of midline-positive $P C$ (B) were compared. A. Higher prostate-specific antigen (PSA) density was the most predictive clinical variable for the presence of PC (area under the ROC curve, 0.797). B. Higher serum PSA concentrations and higher PSA density had similar predictive values for positive midline peripheral zone core biopsies (areas under the ROC curve, 0.881 and 0.898 , respectively).

specimens was higher. Our results indicate that an additional biopsy from the midline PZ is unlikely to be helpful in detecting occult PC.

We added only one core from the midline PZ. Several previous studies used two or three midline cores from the base to the apex. A study characterizing the distribution of prostate carcinoma suggested that nearer to the base, cancer may be more frequent in the lateral aspect, while in the apex, it may be more prevalent in the medial aspect [12]. Our study was directed at the basal portion of the midline $P Z$, and $P C$ may be more frequent in the apical midline PZ. This could explain the absence of patients with only midline PC in our study. The absence of such patients may limit the interpretation of our study. Further investigation is necessary to determine which biopsy level is most effective for the detection of occult midline PC in the absence of other positive cores.

In the subgroup analysis according to prostate volume, transition zone volume, serum PSA concentrations, and PSA density, a higher cancer detection rate was associated with decreased prostate volume and transition zone volume, and increased values of serum PSA concentration and PSA density. These results are consistent with recent studies suggesting that prostate volume [13] and PSA concentration [14] are associated with positive systematic biopsies. Interestingly, the positive midline biopsy rate was highest in the patients with a moderate prostate volume, ranging from $30 \mathrm{~mL}$ to 60 $\mathrm{mL}$. A possible explanation is that a given sample volume might be insufficient to adequately cover a larger prostate volume; therefore, a lower relative sample volume may decrease the sensitivity of the biopsy.

PSA density levels are considered a better predictor of PC than serum PSA concentration alone [6]. In our results, the PSA density was the most predictive indicator for not only the presence of $P C$ but also the presence of midline PC. The $0.15 \mathrm{ng} / \mathrm{mL}^{2}$ cutoff value for the prediction of PC was comparable to the results of a previous study [15]. PSA density demonstrated a better diagnostic performance for midline positive PC, possibly because the midline PC cases in our study were more voluminous and had an increased tumor burden.

Our study had several limitations. First, the retrospective design may have harbored potential selection bias. However, we enrolled all consecutive patients referred from a urology clinic in order to minimize selection bias. Second, false-negatives may have been present in our study population. Not all patients with negative biopsy results received follow-up or underwent repeated biopsy. In our study population, two patients were not diagnosed with $P C$ in a repeated biopsy performed due to clinical suspicion of PC. Although our institution does not perform multiparametric prostate magnetic 
resonance imaging routinely after a negative prostate biopsy, doing so could be helpful in detecting PC if the results of a clinical examination are highly suggestive of PC. It is beyond the scope of our study, but a biopsy under the guidance of multiparametric prostate magnetic resonance imaging might be helpful in detecting PC after a negative routine TRUS-guided biopsy [16]. Finally, the pathologic results of the prostatectomy specimen were not available for all PC patients. Therefore, the exact extent and burden of PC could not be evaluated in our entire study population. However, we did investigate all surgically resected PCs in our study population.

In conclusion, patients showing positive results for $\mathrm{PC}$ in a midline PZ biopsy were found to be more likely to have multiple tumors or large-volume PC with a high tumor burden. Our data indicate that an additional midline core biopsy is unlikely to be helpful in detecting occult midline PC. We do not recommend routine midline core biopsies for the detection of occult midline PCs.

ORCID: Inpyeong Hwang: http://orcid.org/0000-0002-1291-8973; Sang Youn Kim: http://orcid.org/0000-0003-4038-7345; Jeong Yeon Cho: http://orcid.org/00000001-8659-0918; Myoung Seok Lee: http://orcid.org/0000-0001-5285-4874; Seung Hyup Kim: http://orcid.org/0000-0002-9339-5907

\section{Conflict of Interest}

No potential conflict of interest relevant to this article was reported.

\section{Acknowledgments}

This study was supported in part by the Research Fund of the Korean Society of Ultrasound in Medicine.

\section{References}

1. Hodge KK, McNeal JE, Terris MK, Stamey TA. Random systematic versus directed ultrasound guided transrectal core biopsies of the prostate. J Urol 1989;142:71-74.

2. Epstein JI, Walsh PC, Carter HB. Importance of posterolateral needle biopsies in the detection of prostate cancer. Urology 2001:57:1112-1116.

3. Eichler K, Hempel S, Wilby J, Myers L, Bachmann LM, Kleijnen J. Diagnostic value of systematic biopsy methods in the investigation of prostate cancer: a systematic review. J Urol 2006;175:16051612.

4. Chen ME, Troncoso P, Johnston DA, Tang K, Babaian RJ. Optimization of prostate biopsy strategy using computer based analysis. J Urol 1997;158:2168-2175.

5. Guichard G, Larre S, Gallina A, Lazar A, Faucon H, Chemama S, et al. Extended 21-sample needle biopsy protocol for diagnosis of prostate cancer in 1000 consecutive patients. Eur Urol 2007:52:430-435.

6. Stephan C, Stroebel G, Heinau M, Lenz A, Roemer A, Lein $M$, et al. The ratio of prostate-specific antigen (PSA) to prostate volume (PSA density) as a parameter to improve the detection of prostate carcinoma in PSA values in the range of $<4 \mathrm{ng} / \mathrm{mL}$. Cancer 2005;104:993-1003.

7. Epstein Jl, Allsbrook WC Jr, Amin MB, Egevad LL; ISUP Grading Comittee. The 2005 International Society of Urological Pathology (ISUP) Consensus Conference on Gleason Grading of Prostatic Carcinoma. Am J Surg Pathol 2005;29:1228-1242.

8. Harnden P, Naylor B, Shelley MD, Clements $H$, Coles B, Mason MD. The clinical management of patients with a small volume of prostatic cancer on biopsy: what are the risks of progression? A systematic review and meta-analysis. Cancer 2008;112:971-981.

9. Irani J, Blanchet P, Salomon L, Coloby P, Hubert J, Malavaud B, et al. Is an extended 20-core prostate biopsy protocol more efficient than the standard 12-core? A randomized multicenter trial. J Urol 2013; 190:77-83

10. Eskew LA, Bare RL, McCullough DL. Systematic 5 region prostate biopsy is superior to sextant method for diagnosing carcinoma of the prostate. J Urol 1997;157:199-202.

11. Terris MK, Wallen EM, Stamey TA. Comparison of mid-lobe versus lateral systematic sextant biopsies in the detection of prostate cancer. Urol Int 1997;59:239-242.

12. Chen ME, Johnston DA, Tang K, Babaian RJ, Troncoso P. Detailed mapping of prostate carcinoma foci: biopsy strategy implications. Cancer 2000;89:1800-1809.

13. Werahera PN, Sullivan K, La Rosa FG, Kim FJ, Lucia MS, O'Donnell $C$, et al. Optimization of prostate cancer diagnosis by increasing the number of core biopsies based on gland volume. Int J Clin Exp Pathol 2012:5:892-899.

14. Lodeta B, Benko G, Trkulja V. Increasing prostate-specific antigen levels differently influence prostate cancer detection rates of two different 12-core prostate biopsy schemes. Urol Int 2013;91:75-80.

15. Catalona WJ, Richie JP, deKernion JB, Ahmann FR, Ratliff TL, Dalkin $\mathrm{BL}$, et al. Comparison of prostate specific antigen concentration versus prostate specific antigen density in the early detection of prostate cancer: receiver operating characteristic curves. J Urol 1994;152(6 Pt 1):2031-2036.

16. Rastinehad AR, Turkbey B, Salami SS, Yaskiv O, George AK, Fakhoury $M$, et al. Improving detection of clinically significant prostate cancer: magnetic resonance imaging/transrectal ultrasound fusion guided prostate biopsy. J Urol 2014;191:1749-1754. 